\title{
MOLECULAR ANALYSIS OF SUNFLOWER (Helianthus annuus L.) GENOTYPES FOR HIGH OLEIC ACID USING MICROSATELLITE MARKERS
}

\author{
Nagarathna, T.K. , Shadakshari, Y.G. and Ramanappa, T.M.
}

AICRP on Sunflower, University of Agricultural Sciences, GKVK Campus, Bangalore 560065, India

Received: November 15, 2011 Accepted: December 10, 2011

\begin{abstract}
SUMMARY
The sunflower is grown widely for the purpose of edible oil. The oil is most desirable for its oil content and quality because it contains a number of polyunsaturated fatty acids (oleic acid and linoleic acid), as well as saturated fatty acids (palmitic acid and stearic acid). Oils with more unsaturated fatty acids are preferred as they lower the cholesterol content in a human body. High oleic sunflower oil has a higher oxidative stability than most other cooking oils and they are most effective in preventing cardio vascular diseases. Hence, an experiment was conducted with a major objective to evaluate diverse germplasm lines for high oil content and high oleic acid and to identify molecular markers for high oleic acid. The genotypes included $\mathrm{cms}$-lines, R-lines, germplasm lines and inbreds. Initially, the selected genotypes were screened with SSR markers. Among them a few primers showed differentiating bands between low and high oleic contents. To confirm the primer, the initial study was carried out with the released hybrids. Simultaneously, fatty acid profiling was also done for four fatty acids using gas chromatography-mass spectroscopy. Oil content was also estimated in these genotypes by NMR spectrometer. The range for oil content was from $28 \%$ to $44 \%$, whereas for oleic acid it was from $23.63 \%$ to $90.58 \%$. Later the primer was used for further screening of all the genotypes and the parental lines were selected for the heterosis breeding programme.
\end{abstract}

Key words: fatty acid composition, oleic acid, SSR markers, sunflower

\section{INTRODUCTION}

Sunflower, an important oilseed crop, is grown all over the world mainly for the purpose of edible oil. The sunflower seeds contain $35-42 \%$ oil, $20-24 \%$ protein and $4-6 \%$ minerals. Its oil is considered as premium because of its light color, flavour, high smoking point and nutritional quality. The sunflower oil is desirable for

* Corresponding author: nagarathnavijay@rediffmail.com 
HELIA, 34, Nr. 55, p.p. 63-68, (2011)

human consumption due to its favorable fatty acid composition (Baydar \& Erbas, $2005)$. It contains unsaturated fatty acid; linoleic acid (48-74\%), oleic acid (14-40\%) and saturated fatty acid; palmitic acid (4-9\%) and stearic acid (1-7\%) (British Pharmacoparia Commission, 2005). High levels of saturated fat consumption are correlated with increased risk of coronary heart disease. Traditional sunflower oil has saturated fatty acid (SFA) content, which is considered low compared to most vegetables oils. It is necessary to decrease its saturated fat concentration. Oils with high oleic content are resistant to heat oxidation, longer shelf life and low cholesterol effect. A diet in which fat consumption is high in oleic acid is associated with reduced cholesterol, arteriosclerosis, and heart disease (Grundy, 1986; Wardlaw and Snook, 1990; Chang and Huang, 1998). Since consumers are more aware of the quality of oil and amount of fats in their diet, the importance should be put on increasing oil content and improving quality of oils by altering fatty acid composition. This can mainly be done by enhancing oleic acid content.

In order to develop new improved hybrids for high oleic trait, continuous upgrading of genetic base is necessary within the working germplasm through new unexploited lines that may contain desirable traits which can be effectively used in heterosis breeding. But it takes several cycles of crossing and selection to combine the traits of interest with desirable agronomic characters and yield. The use of molecular markers has become an increasingly popular tool for the genetic studies and it is less time-consuming, cheaper, rapid and simple.

Hence, an experiment was conducted with a major objective to evaluate diverse germplasm lines for high oil content and high oleic acid, as well as to identify molecular markers for high oleic acid.

\section{MATERIALS AND METHODS}

\section{Plant material}

For the purpose of screening on high oleic acid, around 350 sunflower genotypes including RHA-lines, $\mathrm{cms}$ lines, inbreds and germplasm lines were planted in February, 2011. Thirty days after sowing, leaves were collected from the field. Genomic DNA was isolated from $100 \mathrm{mg}$ leaf tissue by CTAB method (Doyle, 1987). Concentration of DNA was measured at $260 \mathrm{~nm}$ in a nano spectrophotometer and the quality of DNA was checked by running $5 \mu \mathrm{l}$ of genomic DNA on $0.8 \%$ agarose gel.

\section{Micro satellite analysis}

For the purpose of genotyping the sunflower lines for high oleic content, microsatellite markers were chosen from the patent obtained by Berville et al., 2009. PCR amplification was carried out using $20 \mu \mathrm{l}$ reaction mixture containing $1 \times$ PCR buffer, $0.2 \mu \mathrm{M}$ dNTP's, $0.5 \mathrm{pmol}$ of each primer (forward \& reverse) and 0.2-0.5 
units of Taq polymerase by following the below PCR programme. Amplified PCR products were separated by gel electrophoresis on $1.2 \%$ agarose gel. The touchdown PCR were used for the amplification of the SSR primer as: $94^{\circ} \mathrm{C}$ for $4 \mathrm{~min}, 1$ cycle of $94^{\circ} \mathrm{C}$ for $50 \mathrm{~s}, 56^{\circ} \mathrm{C}$ for $50 \mathrm{~s}, 72^{\circ} \mathrm{C}$ for $1 \mathrm{~min}$. There were 30 cycles. However, the final extension was $2 \mathrm{~min}$ at $72^{\circ} \mathrm{C}$.

Table 1: Primer sequence of SSR primer used for identifying high oleic sunflower lines

\begin{tabular}{ll}
\hline Forward & $5^{`}$ GAGAAGAGGGAGGTGTGAAG 3` \\
Reverse & 5` AGCGGTTATGGTGAGGTCAG 3` \\
\hline
\end{tabular}

\section{Fatty acid profiling}

The seeds were used for the estimation of fatty acids (linoleic acid, oleic acid, palmitic acid and stearic acid) using gas chromatography and the values were expressed in percent. The results were categorized into three groups based on their oleic acid content (low $<25 \%$, medium $26-60 \%$ and high $>60 \%$ ).

\section{RESULTS AND DISCUSSION}

Initial work was carried out with the hybrids released from public and private sectors, while their fatty acid profile was already known (Table 2).

Table 2: Fatty acid profile of some sunflower hybrids (\%)

\begin{tabular}{lccccc}
\hline Hybrids & Palmitic acid & Stearic acid & Oleic acid & Linoleic acid & Oil content \\
\hline KBSH-1 & 5.4 & 4.8 & 51.0 & 38.8 & 38.0 \\
KBSH-44 & 5.6 & 7.3 & 48.9 & 38.2 & 35.4 \\
RSFH-1 & 6.4 & 5.4 & $77.7(\mathrm{HO})$ & 10.5 & 37.0 \\
DRSH-1 & 5.4 & 3.7 & 50.6 & 40.3 & 39.2 \\
PAC-3973 & 4.2 & 7.4 & $82.9(\mathrm{HO})$ & 5.5 & 37.0 \\
VSFH-2042 & 5.1 & 6.9 & $80.4(\mathrm{HO})$ & 7.4 & 38.3 \\
\hline
\end{tabular}

HO: high oleic

Among six hybrids, three were high oleic and three were mid oleic hybrids. Their respective genomic DNA was used for PCR amplification with a primer. The results showed that high oleic containing hybrids (PAC-3973, VSFH-2042 \& RSFH-
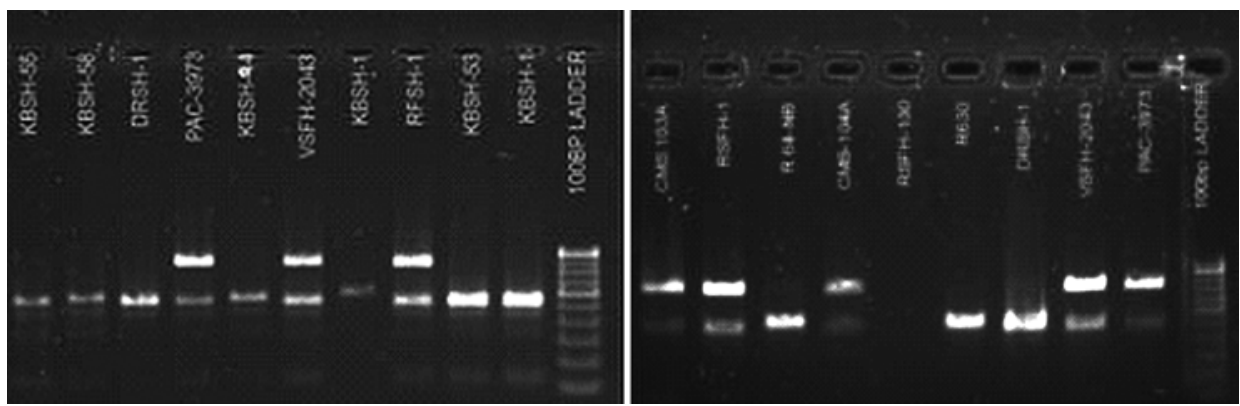

Figure 1: PCR with SSR primer for hybrids and their parental lines 
1) expressed a specific band at 800 to 900 base pair length which was absent in mid oleic hybrids (Figure 1). When the DNA of the hybrid, RSFH-1 along with its parental lines (CMS-103A \& R-64NB) was again amplified with a primer, the female parent (CMS-103A) showed a specific band. The results were confirmed by estimating the fatty acid composition in all the entries tested (Table 3).

Table 3: Fatty acid profiling of sunflower hybrids and their parental lines (values are in percent)

\begin{tabular}{lccccc}
\hline SI no. & Entries & Palmitic acid & Stearic acid & Oleic acid & Linoleic acid \\
\hline 1 & RSFH-1 & 4.40 & 3.16 & 88.20 & 4.24 \\
2 & GKVK-2 & 6.57 & 4.17 & 38.43 & 50.83 \\
3 & $95-\mathrm{C}-1$ & 5.98 & 3.69 & 51.12 & 39.21 \\
4 & KBSH-41 & 7.223 & 3.56 & 31.39 & 57.82 \\
5 & KBSH-1 & 7.09 & 4.59 & 38.62 & 49.70 \\
6 & RHA-6D-1 & 6.50 & 5.17 & 32.32 & 56.01 \\
7 & CMS-17A & 7.17 & 4.53 & 44.57 & 43.72 \\
8 & RHA-23 & 6.01 & 4.77 & 43.91 & 45.31 \\
9 & KBSH-53 & 6.35 & 3.77 & 37.57 & 52.31 \\
10 & KBSH-55 & 5.49 & 4.56 & 53.58 & 36.37 \\
11 & KBSH-58 & 6.22 & 4.84 & 49.49 & 39.45 \\
12 & KBSH-44 & 7.13 & 3.91 & 46.57 & 42.39 \\
13 & R-64-NB & 7.88 & 2.77 & 28.99 & 60.36 \\
14 & CMS-103A & 3.49 & 3.62 & 90.58 & 2.31 \\
15 & CMS-104A & 6.56 & 2.17 & 31.40 & 59.87 \\
16 & RSFH-130 & 7.48 & 2.95 & 33.94 & 55.63 \\
17 & CMS-135A & 6.00 & 4.78 & 53.96 & 35.26 \\
18 & CMS-234A & 5.46 & 5.26 & 60.62 & 28.66 \\
19 & CMS-335A & 7.71 & 3.63 & 23.63 & 65.04 \\
20 & R-630 & 5.58 & 4.23 & 33.19 & 57.00 \\
21 & CMS-851A & 5.36 & 5.21 & 52.39 & 37.04 \\
\hline
\end{tabular}

All these selected entries were analyzed for fatty acid composition using gas chromatography. The genotypes having a specific band showed high oleic content. Furthermore, to identify the parental lines with high oleic acid, genomic DNA of 350 germplasm lines were used for PCR amplification. Simultaneously, the seeds of all entries were used for estimating oil content using nuclear magnetic resonance spectrometer. The range obtained was from $28-44 \%$. Among all the genotypes tested, 8 RHA lines, $16 \mathrm{cms}$ lines and 21 germplasm lines have been identified as high oleic lines (>80\%) with high oil content.

\section{CONCLUSION}

With the marker described by Berville et al., 2009, it is easy to evaluate a huge number of germplasm lines, which helps to identify the high oleic sunflower geno- 
types. Finally the identified RHA lines and cms lines with high oleic acid and high oil content will be used as parental lines in developing new hybrids with high oil content and premium quality of oil.

\section{ACKNOWLEDGEMENT}

The author is thankful to Andre Berville, Montpellier, France for the information on molecular markers in his patent application publication, as well as Rashtriya Krishi Vijnana Yojane, Government of India for funding the research project.

\section{REFERENCE}

British Pharmacopooeia Commission. Ph. Eur. Monograph 1371. British Pharmacopooeia 2005. Norwich, England: The Stationery Office. ISBN 0-11-322682-9.

Hasa Baydar and Sabri Erbas, 2005. Influence of seed development and seed position on oil, fatty acids and total tocopherol contents in sunflower (Helianthus annuus L.). Turk. J. Agric. For. 29: 179-186.

Doyle, J.J. and Doyle, J.L., 1987. A rapid DNA isolation procedure for small quantities of fresh leaf tissue. Phytochem. Bull. 19: 11-15.

Grundy, S.M., 1986. Composition of monounsaturated fatty acid and carbohydrates for lowering plasma cholesterol. New Eng. J. Med. 314: 745-748.

Wardlaw, G.M., Snook, J.T., 1990. Effect of diet high in butter, corn oil, or high-oleic acid sunflower oil on serum lipids and apolipo proteins in men. Am. J. Clin. Nutr. 51: 815-821.

Chang, N.W., Huang, P.C., 1998. Effects of the ratio of polyunsaturated and monounsaturated fatty acid on rat plasma and liver lipid concentration. Lipids 33: 481-487. 
